\title{
ETHzürich
}

\section{Obtaining electron attachment cross sections by means of linear inversion of swarm parameters}

\section{Journal Article}

\section{Author(s):}

Rabie, Mohamed; Haefliger, Pascal; Chachereau, Alise (D); Franck, Christian (D)

Publication date:

2015-02-25

Permanent link:

https://doi.org/10.3929/ethz-b-000097901

Rights / license:

In Copyright - Non-Commercial Use Permitted

Originally published in:

Journal of Physics D: Applied Physics 48(7), https://doi.org/10.1088/0022-3727/48/7/075201 
This is a post-refereeing copy of the manuscript submitted to IOP.

The original version can be found on the IOP website:

Digital Object Identifier: https://doi.org/10.1088/0022-3727/48/7/075201 


\title{
Obtaining electron attachment cross sections by means of linear inversion of swarm parameters
}

\author{
M. Rabie, P. Haefliger, A. Chachereau and C.M. Franck \\ Power Systems and High Voltage Laboratories, ETH Zurich, 8092 Zurich, Switzerland \\ E-mail: cfranck@ethz.ch
}

\begin{abstract}
We investigate electron attachment to strongly attaching gases as a function of the electron energy. The total electron attachment cross section of this electronegative sample gas is determined from the effective ionization rate constant $\nu_{\text {eff }} / N$ measured in a swarm experiment using the buffer gases $\mathrm{N}_{2}$ and $\mathrm{CO}_{2}$ with minor proportions $(\lesssim 0.8 \%)$ of the sample gas $\mathrm{SF}_{6}$, and $\mathrm{C}_{3} \mathrm{~F}_{8}$ respectively. The measured rate constants $\nu_{\text {eff }} / N$ for varying reduced electric field strengths $E / N$ are unfolded from the electron energy distribution of the buffer gases. Different unfolding routines for so-called ill-posed problems are tested to find robust solutions for the attachment cross section. Finally, we propose Tikhonov regularization, which is a well defined algorithm for discrete linear inversion problems, as most efficient. Our method gives rise to an approximate and clearly defined solution for the attachment cross section, and it is described in detail.
\end{abstract}

PACS numbers: 52.80.Dy 51.50.+v 52.25.Fi

Submitted to: J. Phys. D: Appl. Phys.

\section{Introduction}

Electron collision cross sections are required for modeling processes in low temperature plasmas on the basis of the kinetic gas theory [1]. The ability of electronegative gases to attach free electrons is crucial for various industrial applications such as semiconductor manufacturing or high voltage gas insulation [2]. Thus, it is particularly instructive to monitor a molecule's attachment cross section as a function of the incident electron energy. Several methods have been introduced to obtain attachment cross sections and also other types of electron collision cross sections. The most common methods to obtain elastic, excitation or ionization cross sections are ab-initio quantum theoretical calculations [3], electron beam measurements [4] and electron swarm experiments [5].

In the context of swarm experiments, with exquisite control over electrical field strength, density, temperature and mixing ratio of a gas mixture, assessing cross sections is enabled by measuring the swarm parameters [6,7]. The extraction of a full cross section set derived from swarm measurements requires numerical methods for solving 
the Boltzmann equation or Monte Carlo techniques to simulate the motion of electrons. Furthermore, numerical optimization techniques are necessary to adapt an initial guess of cross sections over a wide energy range. In general, these iterative procedures have to deal with the non-uniqueness problem of the obtained cross section sets $[5,7]$.

The attachment cross section $\sigma_{a}$ of a strongly attaching gas can be probed by mixing it in small proportions to a buffer gas, such as $\mathrm{Ar}, \mathrm{N}_{2}$ or $\mathrm{CO}_{2}$, with well known collision cross sections. Then the derivation of $\sigma_{a}$ is decoupled from the entire cross section set of the sample gas, assuming that the mixture is undisturbed by the present sample gas in terms of electron energy distribution. Some methods derive $\sigma_{a}$ by means of algorithms, which minimize the sum of the least squares between calculated and measured effective ionization rate constants $\nu_{\text {eff }} / N$ by iteratively adapting $\sigma_{a}$ towards a positive solution. Such a method "requires a considerable degree of experience and discernment" [8]. Typically, the solution for $\sigma_{a}$ depends on its initial guess as well as on the exact form of the iteration algorithm. Furthermore, the statistical uncertainty of the experimental data affects the outcome.

Extracting cross sections from swarm parameters could be based on a transparent method which gives rise to an approximated and well-defined solution for $\sigma_{a}$. Besides, before trying to unfold the cross section, the actual existence of a usable solution should be checked. Subsequently, if necessary the problem must be modified either experimentally, by e.g. extending the range of measurements, or mathematically by introducing constraints on the solution for the cross section. The latter may also be independent of an initial guess, iteration procedures and measurement noise to make comparison between different data sources possible. Similar to the problem of determining $\sigma_{a}$ from observed rate constants $\nu_{\text {eff }} / N$, in other fields of science and engineering, such as e.g. medical imaging or geophysical prospecting, so called "illposed" problems arise when computing a source that gives rise to some observed data $[9,10,11,12,13]$.

In this work, we adapt the well known linear inversion methods for ill-posed problems to the problem of determining the source $\sigma_{a}$ from the experimentally observed data $\nu_{\text {eff }} / N$. We describe and test our method in detail and compare it with another independent unfolding method, which is based on a gauss function expansion of $\sigma_{a}$. The linear inversion technique is applied to measurements with Sulfur hexafluoride $\mathrm{SF}_{6}$ as well as Octafluoropropane $\mathrm{C}_{3} \mathrm{~F}_{8}$ in the buffer gases $\mathrm{N}_{2}$ and $\mathrm{CO}_{2}$, and the results for $\sigma_{a}$ are compared with literature data. We discuss the limits of this method for unfolding the attachment cross section $\sigma_{a}$ from the rate constants $\nu_{\text {eff }} / N$. Furthermore, we propose the linear inversion method as a simple tool for determining initial guess cross sections for numerical optimization techniques that determine cross section sets from swarm data. 


\section{Theory}

For an equilibrated electron swarm in a binary gas mixture with mixing ratio $k$, the effective ionization rate constant $\nu_{\text {eff }} / N$ is described by [14]:

$$
\nu_{\mathrm{eff}} / N=(1-k) \nu_{\mathrm{eff}}^{\mathrm{B}} / N+k \sqrt{\frac{2}{m_{\mathrm{e}}}} \int_{0}^{\infty}\left(\sigma_{\mathrm{i}}-\sigma_{\mathrm{a}}\right) \varepsilon f \mathrm{~d} \varepsilon .
$$

Here $m_{\mathrm{e}}$ is the electron mass, $\mathrm{N}$ is the number density of the gas. The effective ionization rate constant of the pure buffer gas is $\nu_{\text {eff }}^{\mathrm{B}} / N$, whereas $\sigma_{\mathrm{i}}(\varepsilon)$ and $\sigma_{\mathrm{a}}(\varepsilon)$ are the ionization and attachment cross sections of the sample gas. The electron energy distribution function (EEDF) of the gas mixture is a function of the reduced electric field $E / N$, where $E$ is the electrical field strength. The $\operatorname{EEDF} f(E / N, \varepsilon)$ is normalized to

$$
\int_{0}^{\infty} \sqrt{\varepsilon} f \mathrm{~d} \varepsilon=1
$$

Equation (1) can be written in the form of a Fredholm integral equation of the first kind:

$$
\int_{0}^{\infty} K(E / N, \varepsilon) \sigma_{\mathrm{a}}(\varepsilon) \mathrm{d} \varepsilon=g(E / N)
$$

where the Kernel $K$ is given by the EEDF of the mixture

$$
K(E / N, \varepsilon)=\varepsilon f(E / N, \varepsilon) .
$$

The right-hand side additionally depends on the measured values of $\nu_{\text {eff }} / N$ and $\nu_{\text {eff }}^{\mathrm{B}} / N$ as well as the ionization cross section $\sigma_{\mathrm{i}}$ :

$$
g(E / N)=\int_{0}^{\infty} \sigma_{\mathrm{i}} \varepsilon f \mathrm{~d} \varepsilon+\frac{1}{k} \sqrt{\frac{m_{\mathrm{e}}}{2}}\left\{(1-k) \nu_{\text {eff }}^{\mathrm{B}} / N-\nu_{\text {eff }} / N\right\}
$$

The Kernel $K$ linearly relates the cross section $\sigma_{a}$ with the right-hand side $g$. Furthermore, it is a square integrable function, according to equation (2) and $f(\varepsilon \rightarrow \infty)=0$. This is an important requirement for the linear inversion methods described in section 3.5. If $\nu_{\text {eff }} / N$ and $\nu_{\text {eff }}^{\mathrm{B}} / N$ are measured in the experiment, and the EEDF as well as the cross section $\sigma_{\mathrm{i}}$ are given, then the functions $K$ and $g$ are known. Then, the integral equation (3) corresponds to the classical example of an illposed problem [15]. Its discretization into a matrix problem leads to a system of linear algebraic equations, for which standard routines for the analysis of discrete ill-posed problems can be applied $[16,17]$.

\section{Methods}

\subsection{Pulsed Townsend method}

The experimental method and evaluation procedure applied in this work have been described elsewhere [18]. A short laser pulse of 1.5 ns FWHM releases approximately 
$10^{6}-10^{7}$ start electrons from a photo cathode into the gas volume. The electron swarm drifts in a uniform electric field through a gap between two Rogowski type electrodes. The gap distance is controlled and in the range of 9 to $18 \mathrm{~mm}$ and the applied voltage is up to $40 \mathrm{kV}$. The base pressure is $<10^{-8}$ mbar and the measurements are typically taken between 20-100 mbar and at room temperature. The resulting reduced electrical field strength is typically in the range of $20-200 \mathrm{Td}$, where the upper value is determined by the critical electric field strength that we normally exceed only by a few percent. The displacement current of the electron swarm drifting in the gas gap is recorded and evaluated. From an exponential fit to this current the effective ionization rate $\nu_{\text {eff }}$ is determined, corresponding to the exponential growth/decrease of the electron number in the swarm. The final values for $\nu_{\text {eff }} / N$ and $\nu_{\text {eff }}^{\mathrm{B}} / N$ are obtained by linear regression over the gas number density $N$ :

$$
\nu_{\text {eff }}=\frac{\nu_{\text {eff }}}{N} N \text {. }
$$

Electron attachment to $\mathrm{SF}_{6}$ and $\mathrm{C}_{3} \mathrm{~F}_{8}$ in terms of reaction rates was already investigated previously [19]. In this work, we measure rates for the pure buffer gases $\mathrm{N}_{2}$ and $\mathrm{CO}_{2}$, as well as for mixtures with minor proportions $(\lesssim 0.8 \%)$ of the sample gases $\mathrm{SF}_{6}$ and $\mathrm{C}_{3} \mathrm{~F}_{8}$. The rate constant $\nu_{\text {eff }} / N$ is measured for different reduced field strengths $E / N$, given in Townsend $\left(1 \mathrm{Td}=10^{-21} \mathrm{Vm}^{2}\right)$.

\subsection{Obtaining the kernel $K(E / N, \varepsilon)$}

For solving equation (3), the Kernel $K$ and thus the EEDFs of the gas mixtures are needed, which is in general not known due to non-existing cross section data of the sample gas. Nevertheless, for a buffer gas the change in the electron energy distribution due to a small amount of the sample gas is supposed to be small [20, 21]. This assumption was tested by means of the Boltzmann solver Bolsig + (version 08.2012) using the twoterm approximation [14]. We therefore calculated $\nu_{\text {eff }} / N$ of a gas mixture $\left(0.6 \%\right.$ of $\mathrm{C}_{3} \mathrm{~F}_{8}$ in $\mathrm{Ar}, \mathrm{N}_{2}$ and $\mathrm{CO}_{2}$ ) according to equation (1) using both, the exact EEDF of the mixture and an approximated one, given by the EEDF of the pure buffer gas. The cross section sets from SIGLO database (www.lxcat.net, both retrieved on December 9, 2013) were used. For the buffer gases $\mathrm{N}_{2}$ and $\mathrm{CO}_{2}$, both results yield the same values for $\nu_{\text {eff }} / N$. However, for Ar we detect strong influence of the sample gas on the electron energy distribution and thus on $\nu_{\text {eff }} / N$. For high $E / N$-values, the change of $\nu_{\text {eff }} / N$ due to adding a sample gas might be even dominated by the change of the EEDF and not by the attachment and ionization cross sections of the sample gas.

\subsection{Obtaining the right-hand side $g(E / N)$}

In order to obtain the first term of equation (5), we use the EEDF's $f(E / N, \varepsilon)$ from section 3.2 and reference data for the ionization cross section $\sigma_{\mathrm{i}}$ for $\mathrm{SF}_{6}$ [22] and $\mathrm{C}_{3} \mathrm{~F}_{8}$ [23]. The second term is calculated from the measured $\nu_{\text {eff }} / N$ - and $\nu_{\text {eff }}^{\mathrm{B}} / N$-data (see section 3.1), which is fitted with smoothing spline to evaluate the fit in $1 \mathrm{Td}$ steps. 


\subsection{Discretization}

The discretization of equation (3) into a linear system of equations is necessary to numerically unfold $\sigma_{a}$ on a computer. Two different discretization methods, namely quadrature and expansion methods, are generally used to find approximated solutions for the continuous function $\sigma_{a}$. Expansion methods approximate $\sigma_{a}$ with a set of basis functions, which should be chosen in such a way that they provide a good solution. The subsequent linear inversion would then return the corresponding expansion coefficients. In this work we chose the quadrature method, which evaluates approximated function values at selected abscissas. After the discretization, the linear system of equations is given by $n$ equations and $m$ unknowns, where $n$ is the number of all used EEDF's and $m$ is the length of the solution vector $\sigma_{a}$, which is given by the number of selected abscissas introduced by the quadrature method.

Increasing the number of linear independent algebraic equations for the inversion procedure reduces the noise of the solution for $\sigma_{a}$. Thus, to increase the rank of this Matrix problem, it is instructive to use as many different EEDF's as possible. In the experiment, this would correspond to measuring $\nu_{\text {eff }} / N$ over a wide $E / N$-range for a high variety of buffer gas species.

\subsection{Linear inversion}

The matrix inversion of the discretized form of equation (3) yields a "naive" solution that is very sensitive to perturbation of the right-hand side, which is in our case determined by the measurement error of $\nu_{\text {eff }} / N$ as well as uncertainties in the EEDF. Therefore, to deal with such noise problem, regularization methods that provide stable solution are needed. This fact is mathematically well defined by the so-called discrete Picard condition [17].

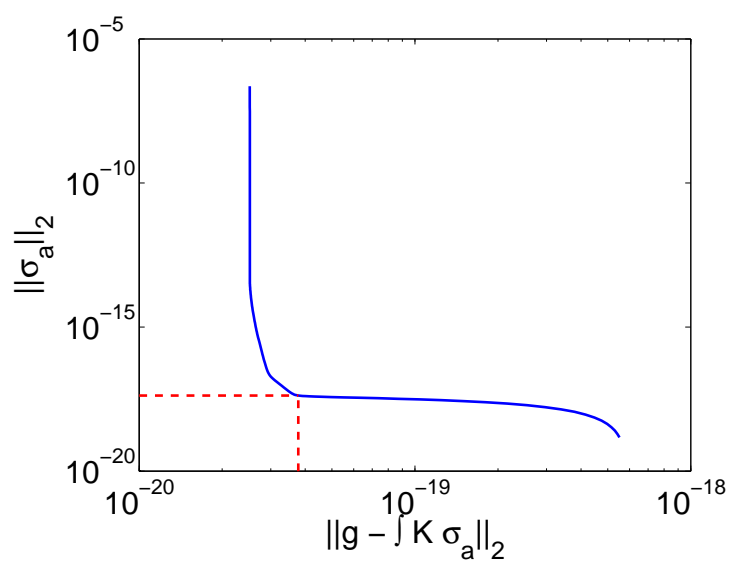

Figure 1. The L-curve for Tikhonov regularization in case of $\mathrm{SF}_{6}$ : logarithmic plot of the solution norm $\left\|\sigma_{\mathrm{a}}\right\|_{2}$ vs. the residual norm $\left\|g-\int_{0}^{\infty} K \sigma_{\mathrm{a}}\right\|_{2}(-)$. The regularization parameter $\lambda$ is tuned from low (upper left) to high (lower right) values, with the regularized $\lambda$-parameter at the corner (- - - ). 
The superior tool for linear inversion is the singular value decomposition (SVD), which is needed for all regularization methods. Common methods are truncated SVD (TSVD), damped SVD (DSVD), compact generalized SVD (CGSVD), Tikhonov regularization or Maximum Entropy regularization, which are all implemented in Matlab Regularization Tools (Version 4.1) [17].

We applied these methods to different measurements involving different kinds of gas compounds, and we identified the Tikhonov regularization as the most convenient method for obtaining attachment cross sections. This method introduces a regularization parameter $\lambda$ that controls the large noise components of the solution by minimizing the functional

$$
\left\|g(E / N)-\int_{0}^{\infty} K(E / N, \varepsilon) \sigma_{\mathrm{a}}(\varepsilon) \mathrm{d} \varepsilon\right\|_{2}+\lambda^{2}\left\|\sigma_{\mathrm{a}}(\varepsilon)\right\|_{2} \longrightarrow 0 .
$$

Depending on the value of $\lambda$, the solution will either be dominated, for small $\lambda$ values, by the perturbations or will be oversmoothed for large $\lambda$-values. To obtain the regularized solution $\sigma_{a}$ for a well defined parameter $\lambda$, the L-curve method is used [17]. It finds a balance between the two norms $\left\|\sigma_{\mathrm{a}}\right\|_{2}$ and $\left\|g-\int_{0}^{\infty} K \sigma_{\mathrm{a}}\right\|_{2}$, which occur in equation (7). Graphically, the L-curve identifies the corner when plotting these norms, as shown in figure 1 for the case of cross section unfolding in $\mathrm{SF}_{6}$, see section 4.1.

If the solution has negative components, we increase $\lambda$ until the absolute value of the minimum is below $5 \%$ of the peak value of the solution. All positive and negative values of $\sigma_{a}$ within this $5 \%$ tolerance are identified as noise and are set to zero.

\subsection{Inversion by gaussian expansion method}

In this subsection we present a gaussian expansion method different from the linear inversion technique in section 3.5. The gaussian expansion method is merely introduced for comparing the results of the linear inversion by an independent method. We do not necessarily propose it as a routine for unfolding cross sections since it is not a direct method, computationally much less efficient than the linear inversion method and it assumes a simplified shape of $\sigma_{a}$.

Nevertheless, in many cases, attachment of electrons in a swarm is dominated by a few channels, such as parent ion attachment and dissociative attachment process. Each channel is typically peaked at a certain energy with a certain width. Therefore, it might be sufficient to approximate the attachment cross sections by two gaussian functions:

$$
\sigma_{a}(\varepsilon) \approx \sum_{i=1}^{2} c_{\mathrm{i}} e^{-\left(\varepsilon-\varepsilon_{\mathrm{i}}\right)^{2} /\left(2 s_{\mathrm{i}}^{2}\right)}
$$

Thus, overall, six parameters are necessary for this simplified model to describe $\sigma_{a}$ : the amplitudes $c_{\{1,2\}}$, widths $s_{\{1,2\}}$ and the positions of the center of the peak $\varepsilon_{\{1,2\}}$. We seek a small number of fit parameters, in order to efficiently minimize the norm $\left\|g-\int_{0}^{\infty} K \sigma_{\mathrm{a}}(\varepsilon) \mathrm{d} \varepsilon\right\|_{2}$. Note that $\sigma_{a}$ is linear in $c_{1,2}$, which therefore can be derived by 
Table 1. Overview of the measurements that were used to determine the attachment cross sections $\sigma_{a}$.

\begin{tabular}{llllll}
\hline & \multicolumn{2}{c}{$\mathrm{SF}_{6}$} & & \multicolumn{2}{c}{$\mathrm{C}_{3} \mathrm{~F}_{8}$} \\
\cline { 2 - 3 } \cline { 5 - 5 } $\begin{array}{l}\text { buffer } \\
\text { gas }\end{array}$ & $\begin{array}{l}\text { mixing } \\
\text { ratio } \%\end{array}$ & $\begin{array}{l}(E / N) \text {-range } \\
(\mathrm{Td})\end{array}$ & & $\begin{array}{l}\text { mixing } \\
\text { ratio } \%\end{array}$ & $\begin{array}{l}(E / N) \text {-range } \\
(\mathrm{Td})\end{array}$ \\
\hline $\mathrm{N}_{2}$ & 0.25 & $95-172$ & 0.81 & $32-150$ \\
& 0.62 & $127-178$ & & \\
\hline $\mathrm{CO}_{2}$ & 0.01 & $11-94$ & 0.27 & $25-104$ \\
& 0.05 & $30-99$ & & \\
\hline
\end{tabular}

linear regression, with the constraint $c_{\{1,2\}} \geq 0$, yielding a positive solution for $\sigma_{a}$. Thus, for the final fit only four independent fit parameters remain, which are $s_{\{1,2\}}$ and $\varepsilon_{\{1,2\}}$

For finding the global minimum, a scatter search algorithm from Matlab MultiStart is used, which uses a great user-chosen number (here we chose $10^{5}$ ) of uniformly distributed start points for the fit parameters within defined bounds [24].

\section{Results}

The attachment cross sections of the two sample gases $\mathrm{SF}_{6}$ and $\mathrm{C}_{3} \mathrm{~F}_{8}$ were unfolded from the measurements summarized in table 1 . The experimental conditions and electron current evaluation procedures are given in section 3.1. The sources of the EEDF's and the ionization cross sections of the sample gases are given in sections 3.2 and 3.3. Following section 3.4 and section 3.5, the linear inversion was applied to the measurement data to find the attachment cross sections $\sigma_{a}$. In addition, the gaussian expansion method was used to find a second independent solution for $\sigma_{a}$.

\subsection{Attachment to $\mathrm{SF}_{6}$}

We investigated $\mathrm{N}_{2} / \mathrm{SF}_{6}$ gas mixtures with $0.25 \%$ and $0.62 \% \mathrm{SF}_{6}$, as well as $\mathrm{CO}_{2} / \mathrm{SF}_{6}$ mixtures with $0.01 \%$ and $0.05 \% \mathrm{SF}_{6}$. The mixing ratios were chosen in such a way that our measured waveforms could still be evaluated over a wide $E / N$-range, especially towards low $E / N$-values. E.g. for $0.05 \% \mathrm{SF}_{6}$ in $\mathrm{CO}_{2}$, the electron current signal vanishes due to the very strong attachment rates below $E / N \approx 30 \mathrm{Td}$, whereas for $0.01 \% \mathrm{SF}_{6}$ in $\mathrm{CO}_{2}$ we can evaluate our signal down to $E / N \approx 11 \mathrm{Td}$. Figure 2 shows the measured rate constants $\nu_{\text {eff }} / N$ for both, (a) $\mathrm{N}_{2}$ and (b) $\mathrm{CO}_{2}$ mixtures with $\mathrm{SF}_{6}$. With increasing $\mathrm{SF}_{6}$ content, $\nu_{\text {eff }} / N$ decreases. We calculated the rate constants by equation (1) with the attachment cross section from linear inversion, gauss expansion as well as from reference data [22], as indicated in figure 2. The results for the unfolded attachment cross section $\sigma_{a}$ are shown in figure 3 and compared with the reference data [22]. 

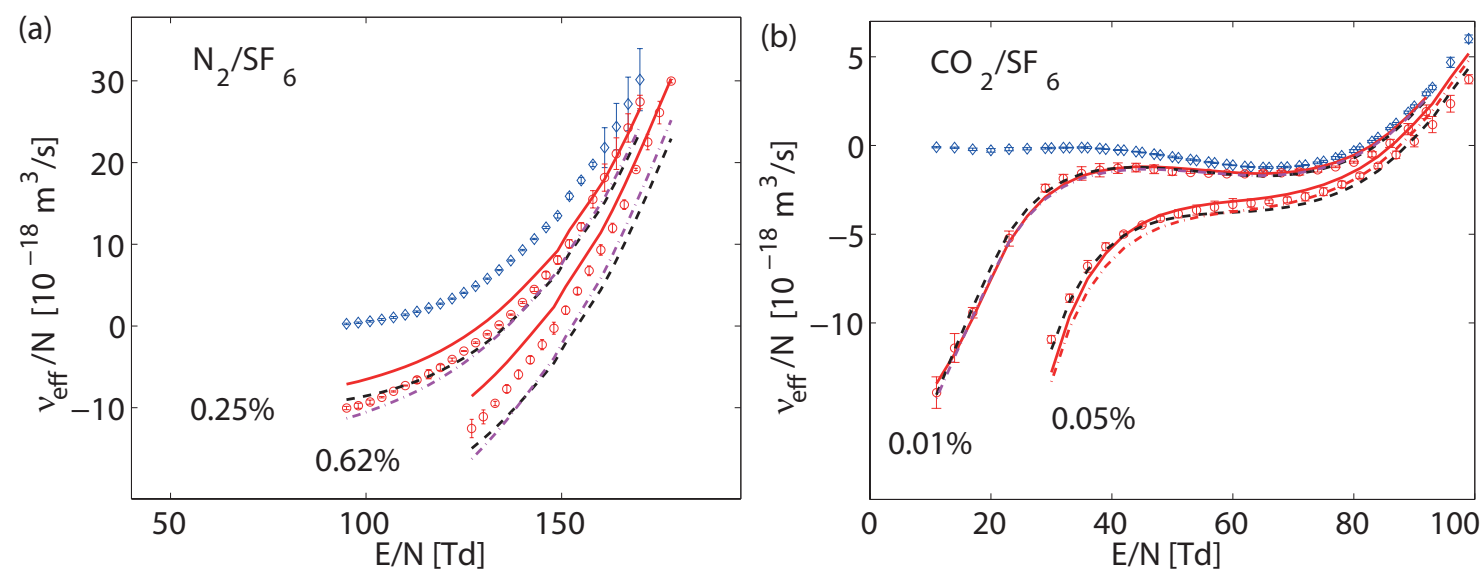

Figure 2. Measured and calculated effective ionization rate constants vs. $E / N$ for $\mathrm{SF}_{6}$ in buffer gases (a) $\mathrm{N}_{2}$ and (b) $\mathrm{CO}_{2}$ : measured $\nu_{\text {eff }} / N$ for pure buffer gas $(\diamond)$ and mixtures $(\mathrm{O})$, as well as calculated values with cross section derived from linear inversion (-), from gaussian expansion (- - -) and from Ref. [22] (- - -).

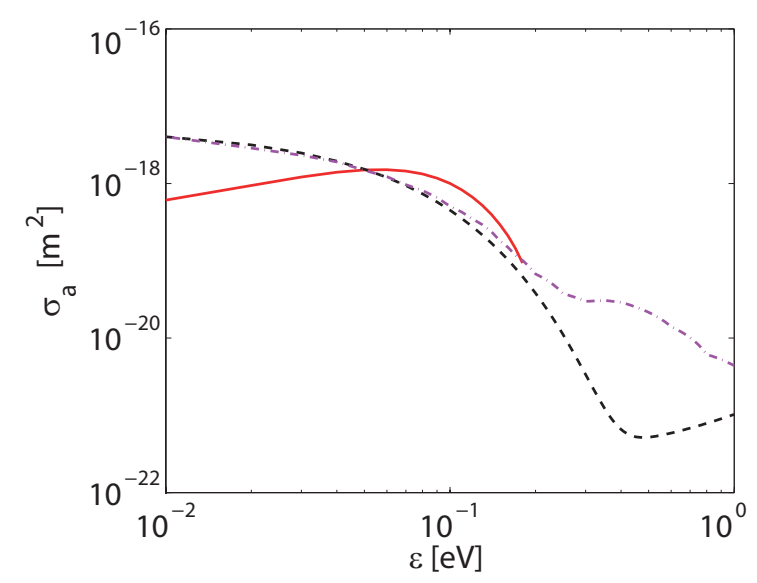

Figure 3. Present results of the attachment cross section of $\mathrm{SF}_{6}$ as a function of electron energy: $\sigma_{\mathrm{a}}$ obtained by means of linear inversion $(-)$ and gaussian expansion (- - - ). For comparison, reference data [22] (- - - ).

\subsection{Attachment to $C_{3} F_{8}$}

We investigated a $\mathrm{N}_{2} / \mathrm{C}_{3} \mathrm{~F}_{8}$ gas mixture with $0.81 \% \mathrm{C}_{3} \mathrm{~F}_{8}$, as well as a $\mathrm{CO}_{2} / \mathrm{C}_{3} \mathrm{~F}_{8}$ mixture with $0.27 \% \mathrm{C}_{3} \mathrm{~F}_{8}$. Figure 4 shows the measured rate constants $\nu_{\text {eff }} / N$ for measurements in (a) $\mathrm{N}_{2}$ and (b) $\mathrm{CO}_{2}$ mixtures. The rate constants calculated by equation (1) with the attachment cross sections obtained from linear inversion, gauss expansion as well as from reference [25] are also indicated in the figure. The measured rate constants decrease by adding $\mathrm{C}_{3} \mathrm{~F}_{8}$ to the buffer gases. The results for the unfolded attachment cross section $\sigma_{a}$ are shown in figure 5 and compared with the reference data [25]. 

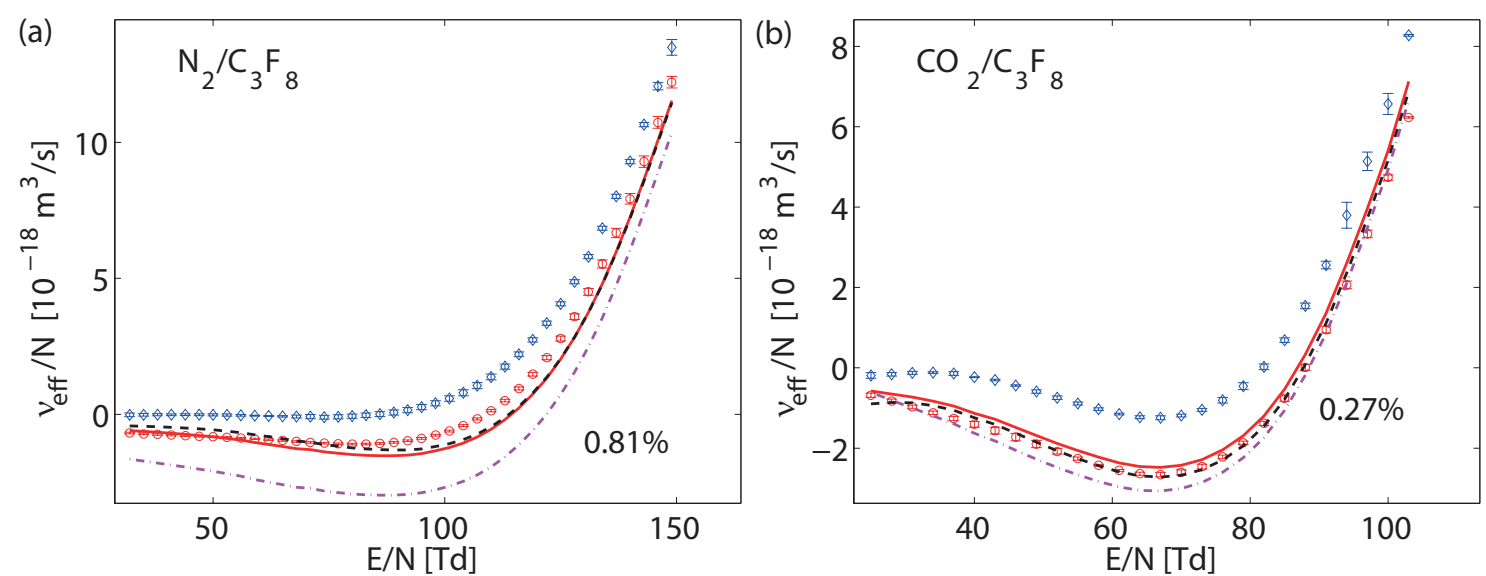

Figure 4. Measured and calculated effective ionization rate constants vs. $E / N$ for $\mathrm{C}_{3} \mathrm{~F}_{8}$ in buffer gases (a) $\mathrm{N}_{2}$ and (b) $\mathrm{CO}_{2}$ : measured $\nu_{\text {eff }} / N$ for pure buffer gas $(\diamond)$ and mixtures $(\mathrm{O})$, as well as calculated values with cross section derived from linear inversion (- $(-)$, from gaussian expansion (- - -) and from Ref. [25] (- - - ).

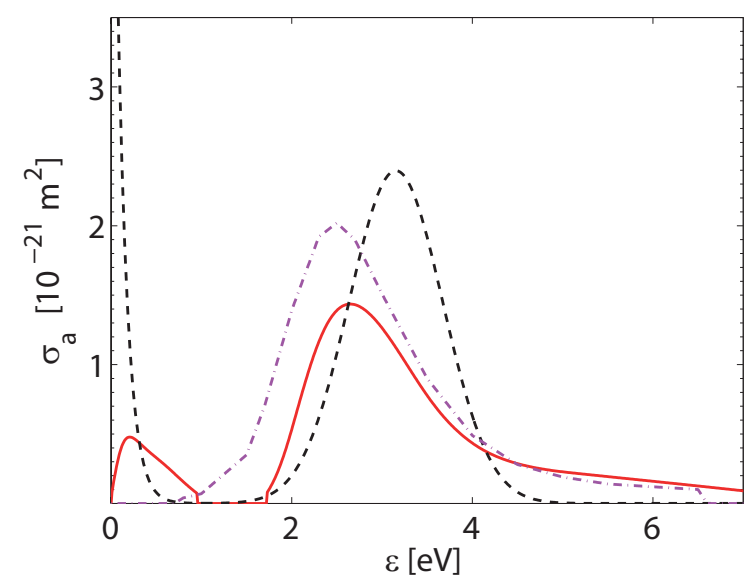

Figure 5. Present results of the attachment cross section of $\mathrm{C}_{3} \mathrm{~F}_{8}$ as a function of electron energy: $\sigma_{\mathrm{a}}$ obtained by means of linear inversion $(-)$ and gaussian expansion (- - - ). For comparison, reference data [25] (- - - ).

\section{Discussion and Conclusion}

\subsection{Thermal electron attachment to $S F_{6}$}

In general, the cross section derived by the linear inversion method is in good agreement with the reference data, see figure 3 . The calculated rate constants fit the measurement $\nu_{\text {eff }} / N$-values well for $\sigma_{a}$ derived by both, linear inversion as well as gaussian expansion, see figure 2. As one can already see by simply comparing the curves, the rate constants calculated by the attachment cross section from Ref. [22] do not fit the measured rate constants distinctly better than the present results.

For energies below $0.01 \mathrm{eV}$, the linear inversion solution for $\sigma_{a}$ tends to converge to zero, whereas electron attachment to $\mathrm{SF}_{6}$ is known to be high near zero energy predominantly due to parent ion attachment [22]. However, the cross section derived by 
means of gaussian expansion is in very good agreement with the reference data in the low-energy range. We tested the linear inversion method with different discretization methods as well as with a higher number of abscissas in order to improve energy resolution. However, the zero-energy peak of $\sigma_{a}$ for $\mathrm{SF}_{6}$ could not be unfolded perfectly from the swarm data. The limiting resolution of swarm derived cross section is generally known and therefore the uncertainty of the swarm-derived attachment cross section for $\mathrm{SF}_{6}$ near zero energy accepted to be large, see e.g. [26]. From a more mathematical perspective, the resolution limit of $\sigma_{a}$ obtained by the linear inversion method, applied to the present $\nu_{\text {eff }} / N$-data, is due to the relatively large $\lambda$-parameter in order to regularize the solution, see section 3.5.

\subsection{Parent ion attachment to $C_{3} F_{8}$}

Electron attachment to $\mathrm{C}_{3} \mathrm{~F}_{8}$ has been investigated in beam experiments [27], which cannot detect the parent ion $\mathrm{C}_{3} \mathrm{~F}_{8}^{-}$due to its reported lifetime of $5 \cdot 10^{-11}-1 \cdot 10^{-8} \mathrm{~s}$ [25]. In swarm studies, pressure dependency due to three body stabilization of shortlived parent anions has been observed for high gas pressures larger than $500 \mathrm{kPa}$ [25].

In contrast to that, we measured a purely linear increase of the rate constant $\nu_{\text {eff }}$ with the gas number density, corresponding to equation (6), without second order contributions $\propto N^{2}$ due to three-body processes. Thus, no significant pressure dependence could be detected over our relatively low pressure range. We conclude that the three-body stabilization does not play a role in our pressure range due to small three-body recombination coefficients for the stabilization of the parent ion.

Both unfolding methods, linear inversion as well as gauss expansion, yield a peak around $3 \mathrm{eV}$ corresponding to dissociative attachment, which is qualitatively in agreement with the reference data [25], see figure 5. Interestingly, in contrast to the same reference, both unfolding methods detect a low-energy contribution to the attachment cross section. The reported lifetimes of $\mathrm{C}_{3} \mathrm{~F}_{8}$ are small compared to the electron transit times of $(1-10) \cdot 10^{-7} \mathrm{~s}$ in our experiment. We conclude from this finding, together with the fact of no observed three body stabilization, that the reported lifetimes for $\mathrm{C}_{3} \mathrm{~F}_{8}$ are at least one order of magnitude larger than reported.

\subsection{Comments on the linear inversion method}

The measurements in $\mathrm{SF}_{6}$ and $\mathrm{C}_{3} \mathrm{~F}_{8}$ give important insights into advantages and problems of the linear inversion method applied to swarm studies. Our results underline the usefulness of the linear inversion method for the investigation of attachment cross sections from $\nu_{\text {eff }} / N$. Nevertheless, several steps in our method have to be questioned carefully. First of all, the method relies on an undisturbed electron energy distribution in the buffer gas when adding a sample gas in small proportion. For the buffer gases $\mathrm{N}_{2}$ and $\mathrm{CO}_{2}$, this assumption seems to be valid as was tested by means of simulations using Bolsig+, whereas in Argon the energy balance of the electrons is affected by collision with the molecules of the sample gas. In general, for the linear 
inversion, too strong attachment rates should be avoided due to the effect of attachment heating [28]. Non-conservative collisions may strongly change the EEDF, which cannot be investigated using Bolsig+, but only via more advanced Monte Carlo methods. Performing measurements with even lower partial pressures of the sample gas can circumvent the problem of a disturbed energy balance.

Secondly, the fact that the inversion problem typically is ill-conditioned and needs regularization might give solutions for $\sigma_{a}$ that are over-smoothed in energy. Often, the regularization parameter has to be increased even more than given by the corner of the L-curve in order to obtain positive solutions for $\sigma_{a}$. In addition, any contribution to $\sigma_{a}$ below a certain threshold, typically $5 \%$ of the peak value, has to be neglected. In case of one dominant attachment channel that exceeds all other weaker channels by at least 1 order of magnitude, only the dominant attachment channel can be detected in the solution of $\sigma_{a} . \mathrm{SF}_{6}$ is such an example where due to very large parent ion attachment, the other channels are not visible within the linear inversion method.

From a more general point of view, the need of regularization might be undesirable since the original inversion problem has to be modified. At the same time, it acts as an indicator for the cross section unfolding method not being accurate without extending the range of the experimental data or any additional information on the shape of the cross section. Therefore, any solution for $\sigma_{a}$ derived from a certain data set by means of an unfolding method that does not regularize the problem is highly questionable being well-defined and robust with regard to uncertainties in the data.

\section{Summary and Outlook}

We introduced a method for the investigation of attachment cross sections from swarm parameters. For this purpose, a sample gas was added in small proportion to different buffer gases and the effective ionization rate constants were measured. The measured data was unfolded from the known electron energy distribution of the pure buffer gas by applying straightforward routines for so-called ill-posed problems in order to obtain an approximated solution for the attachment cross section. We explained the method in detail and applied it to the gases $\mathrm{SF}_{6}$ and $\mathrm{C}_{3} \mathrm{~F}_{8}$. We discussed the usefulness of the method for the cross section derivation from swarm data as well as its limitations.

The rapid determination of the attachment properties of electronegative gases in our experiment gives important insights into their suitability as high voltage insulation gases or as additives in gas mixtures. By our swarm parameter measurements, we aim to investigate the attachment cross sections as well as the critical electric field strengths of interesting candidate gases, for which no complete cross section data exists at this date. 


\section{Acknowledgement}

We thank Heinz Koeppl for valuable support and discussions. This work is financially supported by Alstom (Schweiz) AG, Pfiffner AG and ABB Switzerland.

\section{References}

[1] Robson R E, Nicoletopoulos P, Li B and White R D 2008 Kinetic theoretical and fluid modelling of plasmas and swarms: the big picture, Plasma Sources Science and Technology 17024020

[2] Christophorou L G and Olthoff J K 2002 Electron interactions with plasma processing gases: present status and future needs Applied Surface Science 192309

[3] Huo W M, Gianturco F A 1995 Computational Methods for Electron-Molecule Collisions Plenum, New York

[4] Christophorou L G (Ed.) 1984 Electron-molecule interactions and their applications Vol. 1 Academic Press

[5] Huxley L G H and Crompton R W 1974 The diffusion and drift of electrons in gases Wiley, New York

[6] Morgan W L 2000 Electron collision data for plasma chemistry modeling Adv. At. Mol. Opt. Phys. 4379

[7] Petrovic Z Lj, Dujko S, Maric D, Malovic G, Nikitovic Z, Sasic O, Jovanovic J, Stojanovic V and Radmilovic-Radenovic M 2009 Measurement and interpretation of swarm parameters and their application in plasma modelling J. Phys. D: Appl. Phys. 42194002

[8] Christophorou L G, McCorkle D L, Anderson V E 1971 Swarm-determined electron attachment cross sections as a function of electron energy J. Phys. B: Atom. Molec. Phys. 41163

[9] Parker R L 1977 Understanding inverse theory Ann. Rev. Earth Planet Sci. 5 35-64

[10] Tikhonov A N and Goncharsky A V 1987 Ill-Posed Problems in the Natural Sciences MIR Publishers, Moscow

[11] Natterer F 1986 The Mathematics of Computerized Tomography John Wiley, New York

[12] Colton D and Kress R 1983 Integral Equation Methods for Scattering Theory John Wiley, New York

[13] Craig I J D, Brown J C 1986 Inverse Problems in Astronomy Adam Hilger, Bristol

[14] Hagelaar G J M, Pitchford L C 2005 Solving the Boltzmann equation to obtain electron transport coefficients and rate coefficients for fluid models Plasma Sources Sci. Technol. 14 722-733

[15] Groetsch C W 1984 The Theory of Tikhonov Regularization for Fredholm Equations of the First Kind Pitman, Boston

[16] Hansen P C 2010 Discrete Inverse Problems: Insight and Algorithms Fundamentals of Algorithms, SIAM

[17] Hansen P C 1994 Regularization Tools: A Matlab package for analysis and solution of discrete ill-posed problems Numerical Algorithms 6 1-35

[18] Dahl D A, Teich T H and Franck C M 2012 Obtaining precise swarm parameters from a pulsed Townsend setup J. Phys. D: Appl. Phys. 45485201

[19] Dahl D A and Franck C M 2013 Response analysis of electron attachment rates to $\mathrm{C}_{3} \mathrm{~F}_{8}$ and $\mathrm{SF}_{6}$ in buffer gases J. Phys. D: Appl. Phys. 46445202

[20] Blevin H A, Fletcher J and Hunter S R 1985 Electron-velocity distribution functions in gases: The influence of anisotropic scattering and electron nonconservation by attachment and ionization Phys. Rev. A 312215

[21] Bordage M C, Segur P and Chouki A 1996 Determination of a set of electron impact cross sections in tetrafluoromethane consistent with experimental determination of swarm parameters $J$. Appl. Phys $\mathbf{8 0} 1325$ 
[22] Phelps A V and Van Brunt R J 1988 Electron transport, ionization, attachment, and dissociation coefficients in SF6 and its mixtures J. Appl. Phys. 644269

[23] Christophorou L G and Olthoff J K 1998 Electron interactions with C3F8 J. Phys. Chem. Ref. Data 27889

[24] Ugray Z, Lasdon L S, Plummer J C, Glover F, Kelly J P, Mart R 2007 Scatter Search and Local NLP Solvers: A Multistart Framework for Global Optimization INFORMS Journal on Computing 19328

[25] Hunter S R and Christophorou L G 1984 Electron attachment to the perfluoroalkanes $n-\mathrm{C}_{n} \mathrm{~F}_{2 N+2}$ $(N=1-6)$ using high pressure swarm techniques J. Chem. Phys. 806150

[26] Christophorou L G and Olthoff J K 2000 Electron interactions with SF6 J. Phys. Chem. Ref. Data 29267

[27] Spyrou S M, Sauers I and Christophorou L G 1983 Electron attachment to the perfluoroalkanes $\mathrm{n}_{-} \mathrm{C}_{N} \mathrm{~F}_{2} N+2 N=1-6$ and $\mathrm{i}-\mathrm{C}_{4} \mathrm{~F}_{1} 0$ J. Chem. Phys. 787200

[28] Robson R E and Ness K F 1986 Velocity distribution function and transport coefficients of electron swarms in gases: Spherical-harmonics decomposition of Boltzmann's equation Phys. Rev. A 33 2068 